\title{
(6)
}

\section{Existe associação entre periodontite e hipertensão arterial?}

Carlos Medeiros Malcher (1)

\section{REVISÃO DE LITERATURA}

Resumo

Estima-se que a periodontite afete mais de $50 \%$ da população mundial e, em sua forma grave, é a sexta doença humana mais comum. Evidências clínicas apoiam que a periodontite afeta a função endotelial sistêmica e isso pode ter um impacto na hipertensão; alguns relatos sugerem possiveis efeitos diretos da bacteriemia oral relacionada à microbiota também na disfunção vascular. $O$ objetivo do presente artigo é compreender a patogênese da hipertensão e sua associação com a periodontite, bem como os possíveis mecanismos comuns envolvidos. Foi realizada uma revisão da literatura, por meio de consulta ao MEDLINE, a fim de analisar dados recentes que sustentam que pacientes com periodontite têm maior probabilidade de apresentar hipertensão arterial. A estrutura conceitual que apoia essa hipótese é discutida. Os dados corroboram que, na presença de periodontite moderada, a probabilidade de ter hipertensão é de $22 \%$ e essa probabilidade aumenta para $49 \%$ nas formas graves. Essa condição pode contribuir potencialmente para a imunidade próhipertensiva de várias maneiras; além disso, pode estar associado a disfunção endotelial permanente. Assim, tornou-se evidente que a inflamação e o sistema imunológico têm um papel causal na patogênese da hipertensão, embora esse mecanismo ainda não esteja claro. Da nossa análise podemos concluir que existe uma associação entre periodontite e hipertensão e, portanto, a terapia periodontal pode ser considerada como uma ferramenta na prevenção e tratamento da hipertensão. O desafio ainda está aberto para pesquisas direcionadas ao entendimento da patogênese da hipertensão.

Palavras-chave: Pressão sanguínea, Hipertensão arterial, Estresse oxidativo. 


\title{
Is there an association between periodontitis and arterial hypertension?
}

\begin{abstract}
Periodontitis is estimated to affect more than $50 \%$ of the world's population and, in its severe form, it is the sixth Most common human disease. Clinical evidence supports that periodontitis affects systemic endothelial function and this may have an impact on hypertension; some reports suggest possible direct effects of microbiota-related oral bacteremia also on vascular dysfunction. The aim of this article is to understand the pathogenesis of hypertension and its association with periodontitis, as well as the possible common mechanisms involved. A review of the literature was carried out, by consulting MEDLINE, in order to analyze recent data that support that patients with periodontitis are more likely to have arterial hypertension. The conceptual framework that supports this hypothesis is discussed. The data corroborate that, in the presence of moderate periodontitis, the probability of having hypertension is $22 \%$ and this probability increases to $49 \%$ in severe forms. This condition can potentially contribute to prohypertensive immunity in several ways; in addition, it may be associated with permanent endothelial dysfunction. Thus, it became evident that inflammation and the immune system play a causal role in the pathogenesis of hypertension, although this mechanism remains unclear. From our analysis we can conclude that there is an association between periodontitis and hypertension and, therefore, periodontal therapy can be considered as a tool in the prevention and treatment of hypertension. The challenge is still open for research aimed at understanding the pathogenesis of hypertension.
\end{abstract}

Keywords: Blood pressure, High blood pressure, Oxidative stress.

Instituição afiliada: 1- Professor da graduação em Odontologia da Faculdade de Fortaleza, Fortaleza Ceará - Brasil.

Dados da publicação: Artigo recebido em 03 de julho, revisado em 11 de julho, aceito para publicação em 20 de julho e publicado em 30 de julho.

DOI: https://doi.org/10.36557/2674-8169.2021v3n7p03-10

Carlos Medeiros Malcher c.rod.334@gmail.com

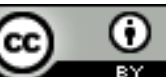

This work is licensed under a Creative Commons Attribution 4.0 International License. 


\section{INTRODUÇÃO}

A hipertensão arterial, definida como tendo valores de $\geq 140 \mathrm{mmHg}$ pressão arterial sistólica e / ou $\geq 90 \mathrm{mmHg}$ pressão arterial diastólica (1), afeta 30-45\% da população adulta e está intimamente associada a eventos cardiovasculares adversos, como acidente vascular cerebral, infarto do miocárdio, morte súbita, insuficiência cardíaca, doença arterial periférica e insuficiência renal. Por outro lado, a periodontite afeta mais de $50 \%$ da população mundial, com incidência ainda maior em países de baixa e média renda (2). Vários estudos analisaram a relação entre doenças periodontais e hipertensão arterial. Ao recuperar o MedLine [hipertensão arterial + periodontite e / ou doença periodontal; Abril 2020], encontramos cerca de 680 registros correspondentes, entre eles, apenas 10 são ensaios clínicos em humanos e 12 são revisões sistemáticas dos últimos 5 anos.

Entre as revisões sistemáticas, uma meta-análise recente de todos os estudos disponíveis (3) apóia uma significativa "sobreposição" entre essas duas condições: entre os indivíduos que sofrem de doença gengival grave, os hipertensos representariam $49 \%$. Na verdade, a pressão arterial média foi encontrada para ser maior em 4,5 mm Hg para PAs e $2 \mathrm{mmHg}$ para PAD em pacientes afetados com periodontite do que naqueles que não eram; esses dados são compatíveis com a evidência que apóia um aumento de $25 \%$ no risco de morte por ataque cardíaco ou acidente vascular cerebral devido a um aumento de PAs de $5 \mathrm{mmHg}$ (4).

A hipertensão arterial é considerada um mecanismo complexo e multifatorial que inclui disfunção endotelial, estresse oxidativo, inflamação e resposta imune; de fato, todos esses fatores são capazes de alterar o controle neuro-humoral da pressão arterial (5). No entanto, o entendimento da patogênese da hipertensão ainda é incompleto. Por outro lado, a periodontite é uma doença inflamatória multifatorial crônica causada por patógenos invasivos que alteram a microbiota e o microbioma oral, levando a uma destruição progressiva dos tecidos de suporte do dente com instabilidade / perda de dentes e um impacto negativo na qualidade de vida do paciente (6). O objetivo deste artigo é analisar as evidências que associam periodontite e hipertensão em humanos e o possível mecanismo dessa associação.

\section{Perturbação da homeostase microbiana}

A inflamação periodontal inicia uma inflamação sistêmica e o monitoramento de marcadores inflamatórios como a proteína $C$ reativa ou os níveis de fibrinogênio pode ser útil para sua detecção (7). A interação entre a bactéria e o hospedeiro é o mecanismo 
biológico mais plausível que conecta a periodontite a uma série de doenças sistêmicas crônicas, como doenças respiratórias, doenças pulmonares obstrutivas crônicas e doenças cardíacas coronárias, bem como eventos relacionados a doenças cardíacas coronárias, como como angina e infarto, aterosclerose, acidente vascular cerebral, diabetes mellitus, trabalho de parto prematuro e parto com baixo peso ao nascer (8).

Microbiota é um agregado de microorganismos que colonizam e residem em ou dentro de tecidos e biofluidos humanos; O microbioma, por outro lado, indica a totalidade do patrimônio genético pertencente à microbiota. É, portanto, evidente que o microbioma se refere a todas as expressões gênicas.

A possível ligação entre inflamação crônica e hipertensão e ativação imune próhipertensiva é explicada na figura 1.
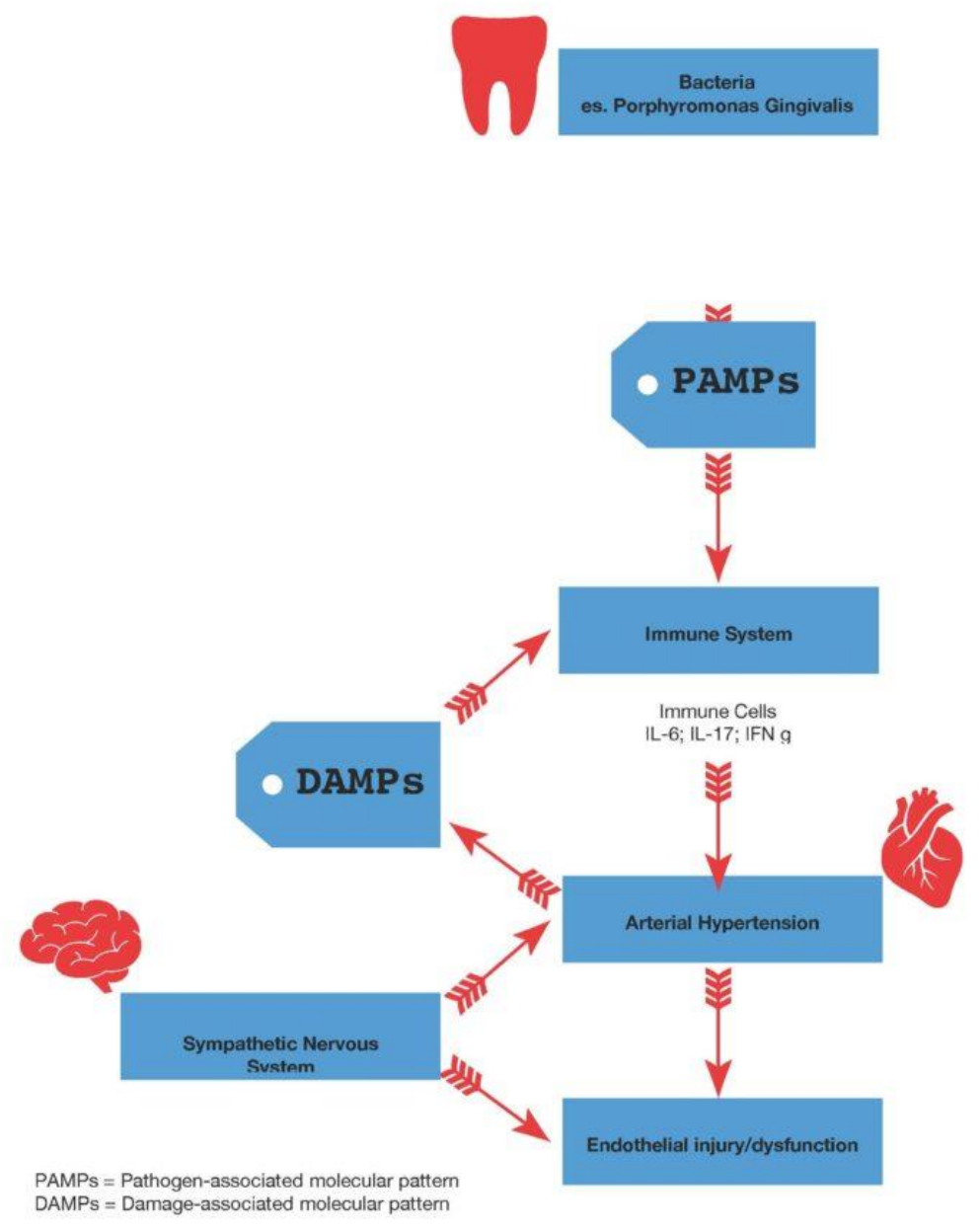

Figura $1 \mathrm{~A}$ possível conexão entre inflamação crônica e hipertensão e ativação imune pró-hipertensiva.

\section{Hipertensão e periodontite}


A causa da hipertensão é uma questão sem solução. Para responder a essa questão, a análise percorreu dois caminhos: o primeiro examinou as interferências genéticas e o segundo se beneficiou de resultados de ensaios clínicos randomizados. Estudos recentes de associação do genoma em grande escala identificaram quatro polimorfismos que foram associados à periodontite. A randomização mendeliana foi usada e esse método identificou variantes pleiotrópicas horizontais e mostrou influência significativa da periodontite em todos os valores de pressão arterial, sistólica, diastólica e média. Para confirmar a associação entre doença periodontal e hipertensão, um ensaio clínico randomizado foi realizado sobre os efeitos da terapia periodontal na hipertensão; foi observado que a monitorização ambulatorial da pressão sistólica e diastólica por 24 horas em pacientes tratados com tratamento periodontal intensivo resultou em uma redução significativa da pressão arterial sistólica e diastólica. Além disso, a inflamação sistêmica medida pelo nível de citocinas circulantes (IFN-g, IL-17A, TNF- $\alpha$, IL-6) diminuiu significativamente em pacientes submetidos à terapia periodontal intensiva (9).

Sabemos que na patogênese da doença periodontal, citocinas produzidas por células da resposta imune inata, incluindo TNF- $\alpha$, IL-1b e IL-6, são as primeiras a serem secretadas após o reconhecimento microbiano (10). Bactérias e mediadores inflamatórios podem entrar no sangue e se espalhar sistemicamente com um impacto mensurável na inflamação sistêmica (11). Usando técnicas de amplificação de DNA, o DNA bacteriano periodontal foi detectado no ateroma de carótida (12). A doença periodontal não é universalmente expressa em indivíduos com má higiene oral, a expressão da doença também requer um hospedeiro sensível (13).

\section{CONCLUSÃO}

Estima-se que a periodontite afete mais da metade da população mundial e, em sua forma grave, é a sexta doença humana mais comum. Como afirmado acima, a evidência clínica apóia a noção de que a periodontite afeta a função endotelial sistêmica e isso pode ter um impacto na hipertensão; alguns relatos sugerem possíveis efeitos diretos da bacteremia oral relacionada à microbiota também na disfunção vascular.

A associação postulada entre periodontite e hipertensão foi verificada como estatisticamente significativa, portanto, uma possível indicação é que a terapia periodontal pode ser instrumental na prevenção e tratamento da hipertensão.

Concluindo, é necessária uma mudança de paradigma e devemos começar a considerar a saúde bucal uma prioridade global. O desafio para a investigação nesta área consiste, portanto, em compreender os mecanismos patogenéticos que configuram essas relações e, no domínio da medicina personalizada, informar os doentes com doença periodontal sobre o risco de ter / desenvolver hipertensão e aconselhar a saúde oral em. pessoas já afetadas. Em pacientes que não sabem que têm pressão alta, uma forma simples poderia ser medir a pressão arterial antes da consulta ao dentista. 


\section{THE AUTHORS DECLARE NO CONFLICTS OF INTEREST.}

\section{REFERÊNCIAS}

1. Williams B, Mancia G, Spiering W, Agabiti Rosei E, Azizi M, Burnier M, Clement DL, Coca A, de Simone G, Dominiczak A, Kahan T, Mahfoud F, Redon J, Ruilope L, Zanchetti A, Kerins M, Kjeldsen SE, Kreutz R, Laurent S, Lip GYH, McManus R, Narkiewicz K, Ruschitzka F, Schmieder RE, Shlyakhto E, Tsioufis C, Aboyans V, Desormais I; Authors / Task Force Members. 2018 ESC / ESH Guidelines for the management of arterial hypertension: the Task Force for the management of arterial hypertension of the European Society of Cardiology and the European Society of Hypertension. J Hypertens 2018; 36: 1953-2041.

2. Peres MA, Macpherson LMD, Weyant RJ, Daly B, Venturelli R, Mathur MR, Listl S, Keller Celeste R, Guarnizo-Herre no CC, Kearns C, Benzian H, Allison P, Watt RG. Oral diseases: a global public health challenge. Lancet 2019;394:249-260.

3. Muñoz Aguilera E, Suvan J, Buti J, Czesnikiewicz-Guzik M, Barbosa Ribeiro A, Orlandi M, Guzik TJ, Hingorani AD, Nart J, D'Aiuto F. Periodontitis is associated with hypertension: a systematic review and meta-analysis. Cardiovasc Res. 2020;116(1):28-39.

4. Holtfreter B, Empen K, Gläser S, et al. Periodontitis is associated with endothelial dysfunction in a general population: a cross-sectional study. PLoS One. 2013;8(12):e84603.

5. Drummond GR, Vinh A, Guzik TJ, Sobey CG. Immune mechanisms of hypertension. Nat Rev Immunol 2019;19:517-532.

6. Buset S, Walter C, Friedmann A, Weiger R, Borgnakke WS, Zitzmann NU. Are periodontal diseases really silent? A systematic review of their effect on quality of life. J Clin Periodontol 2016;43:333.

7. Slade GD, Ghezzi EM, Heiss G, Beck JD, Riche E, Offenbacher S. Relationship between periodontal disease and C-reactive protein among adults in then Atherosclerosis Risk in Communities study. Arch Intern Med. 2003;163(10):1172-9.

8. Saini R, Marawar PP, Shete S, Saini S. Periodontitis, a true infection. J Glob Infect Dis. 2009;1(2):149-150.

9. Czesnikiewicz-Guzik M, Osmenda G, Siedlinski M, Nosalski R, Pelka P, Nowakowski D, Wilk G, Mikolajczyk TP, Schramm-Luc A, Furtak A, Matusik P, Koziol J, Drozdz M, MunozAguilera E, Tomaszewski M, Evangelou E, Caulfield M, Grodzicki T, D’Aiuto F, Guzik TJ. Causal association between periodontitis and hypertension: evidence from Mendelian randomization and a randomized controlled trial of non-surgical periodontal therapy. Eur Heart J. 2019 Nov 1;40(42):3459-3470. 
10. Garlet GP. Destructive and protective roles of cytokines in periodontitis: a re-appraisal from host defense and tissue destruction viewpoints. J Dent Res. 2010 Dec;89(12):134963.

11. Van Dyke TE, van Winkelhoff AJ. Infection and inflammatory mechanisms. J Clin 2013 Apr;40 Suppl 14:S1-7.

12. Haraszthy VI, Zambon JJ, Trevisan M, Zeid M, Genco RJ. Identification of periodontal pathogens in atheromatous plaques. J Periodontol. 2000 Oct;71(10):1554-60. PubMed PMID: 11063387.

13. Barros SP, Hefni E, Nepomuceno R, Offenbacher S, North K. Targeting epigenetic mechanisms in periodontal diseases. Periodontol 2000. 2018;78(1):174-184.

14. Ciulla, M., and Patrizia Vivona. "Is there an association between periodontitis and arterial hypertension." Ital J Dent Med 5 (2020): 17-9.

\section{DECLARAÇÃO CCBYNC}

Este artigo é uma cópia com adaptação para o português do original "Ciulla, M., and Patrizia Vivona. "Is there an association between periodontitis and arterial hypertension." Ital J Dent Med 5 (2020): 17-9." (14)

Esta cópia com adaptação para o português teve como alteração além do idioma, a autoria do artigo e lyout do PDF.

Os autores da publicação original não deram endosso específico a esta cópia com adaptação para a português, a não ser a licença CCBYNC 4.0 disponibilizada pelo periódico que publicou o artigo original.

Este é o link da licença: https://creativecommons.org/licenses/by/4.0/

Este é o link do artigo original: http://www.dentalmedjournal.it/is-there-an-associationbetween-periodontitis-and-arterial-hypertension/ 